\title{
MORPHOMETRIC PARAMETERS OF CLAVICLES AMONG ADULT BLACK PEOPLE IN TANZANIA.
}

\section{Alexander AG, Russa AD}

Correspondence to Dr. A.D. Russa, Department of Anatomy, School of Medicine, Muhimbili University of Health and Allied Sciences, PO Box 65001, Dares Salaam, Tanzania. Email: adrussa@yahoo.com // drussa@muhas.ac.tz. Tel: +255755 524771

Fax: +255222150465

\begin{abstract}
Morphological clavicular variation has been studied extensively by orthopaedic surgeons, anatomic and forensic experts to explain developmental, gender and age-related differences. The design of fixation devices for displaced clavicular fracture management depends largely on anatomic characteristics of clavicle. Eighty-one unpaired clavicles of unknown sex were studied, 42 clavicles were of right side and 39 clavicles of left side. All the clavicles were collected from adult cadavers which were dissected in Departments of Anatomy at Muhimbili and Herbert Kairuki Memorial Universities. The length of clavicle was measured by a vernier calliper; the middle point of this length was taken as the point where midclavicular circumference was measured with the help of a measuring thread and the angle of curvature of clavicle was measured by using protractors. The average lengths of the left and right clavicles were $15.23 \pm 1.12 \mathrm{~cm}$ and $15.43 \pm 1.01 \mathrm{~cm}$ respectively. The average medial angle of curvature of left clavicle was $155.33^{\circ} \pm 4.39^{\circ}$ and that of right clavicle was $153.40^{\circ} \pm 3.96^{\circ}$. The mean total angle of curvature of left clavicle was $293.54^{\circ} \pm 9.55^{\circ}$ and the average total angle of curvature of right clavicle was $290.05 \pm 8.94^{\circ}$. The average midclavicular circumference of left clavicle was $3.88 \mathrm{~cm} \pm 0.33 \mathrm{~cm}$ and that of right clavicle was $3.94 \mathrm{~cm} \pm 0.33 \mathrm{~cm}$. The right clavicle was longer than the left clavicle, the average medial angle of curvature of left clavicle was greater than medial angle of right clavicle, the average lateral angle of curvature of left clavicle was more than the average lateral angle of curvature of right clavicle and the mean of midclavicular circumference of right clavicle was greater than that of left clavicle.
\end{abstract}

Keywords: Morphometric, clavicle, curvatures, circumference.

\section{INTRODUCTION}

Clavicular morphology has been a subject of interest for researchers for a long time. This has been studied extensively by orthopaedic surgeon for better management of clavicular fractures, anatomic and forensic experts have studied clavicle to explain development, gender and age related differences (Rathna, 2015). This paired long bone placed horizontally with shaft and two ends has double curvature of shaft which separates the clavicle into medial two third and lateral one third. The medial two-third of the shaft is rounded and has four surfaces. The anterior surface is convex forward; this provides space for the neurovascular bundle of upper limb and the posterior surface is smooth. The superior surface is rough in its medial part and the inferior surface has a rough oval impression at the medial end (Kaur et al., 2002, Alcina et al., 2015). The lateral half of the later has a longitudinal subclavian groove (Ishwarkumar et al., 2016). Human clavicle fractures account for $3 \%-10 \%$ of all fractures and for $35-44 \%$ of fractures about the shoulder (Qiu et al., 2016). Middle-third fractures account for $80 \%$ of all clavicular fractures; whereas, fractures of the lateral and medial third of the clavicle account for $15 \%$ and $5 \%$, respectively (Bernat et al., 2014). Although clavicular fracture is treated 
conservatively; there is an increase in incidence of non-union as well as displaced fractures with conservative treatment (Alcina et al., 2015). Fixation of clavicular fracture has shown improved benefits over the conservative methods, several studies have reported poor outcome after conservative treatment of severely displaced midclavicular fractures resulted in shoulder shortening, non-union, or function deficits (Hillen et al., 2016). The design of fixation devices depends largely on anatomic characteristics of clavicle (Liu et al., 2010).

There is evidence from past studies that the length of left clavicle is longer than that of right clavicle. The design of fixation devices depends largely on anatomic characteristics of clavicle. Those characteristics include the length of clavicle, angle of curvature of clavicle and the mid clavicular circumferences. In order to do intermedullary nailing of clavicle a physician needs to know the whole morphometric parameters of clavicle of a given population (Aira et al., 2017). Little is known on Tanzanians morphometric parameters of clavicles. This study is filling that gap. Previous studies show that there are morphological differences in term of length; clavicular length has influence on its dimensions and they will vary with respect to gender, side and geographical factors (Rathna, 2015), mid-clavicular circumference and angle of curvatures of clavicles among human beings, right and left clavicular morphological parameters from the same individual are also different. There is scarcity of data in Tanzania concerning morphological parameters of clavicles as no study has been done. Data from this study will be helpful by giving a clear current situation on existing variation among black people of Tanzania.

\section{MATERIAL AND METHODS:}

The descriptive cross-sectional study design was used. Eighty-one unpaired clavicles of unknown sex, forty-two clavicles were of right side and 39 clavicles of left side. All clavicles were collected from adult cadavers which were dissected in department of Anatomy at Muhimbili University of health and allied science and Hubert Kairuki Memorial University. The length of clavicle was measured by the vernier calliper ignoring the curve of the clavicle and was recorded in centimetre. The middle point of this length was taken as the point where mid-clavicular circumference was measured with the help of measuring thread and was recorded in centimetre. The angle of curvature of clavicle was measured by using protractors. The data was subjected to statistical analysis.

All clavicles found in department of anatomy which were dissected from cadavers and bone showing any defects was excluded from the study. Total of 81 clavicles were collected and used during this study. The data list was used to collect data. The data obtained were recorded in table of results and analysis of data was done by using SPSS and p-value $\leq 0.05$ was considered to statistically significant. Permission to conduct the study was sought from Ethical Clearance Committee of MUHAS and from administrative authorities of all the medical universities from which data collection were conducted 


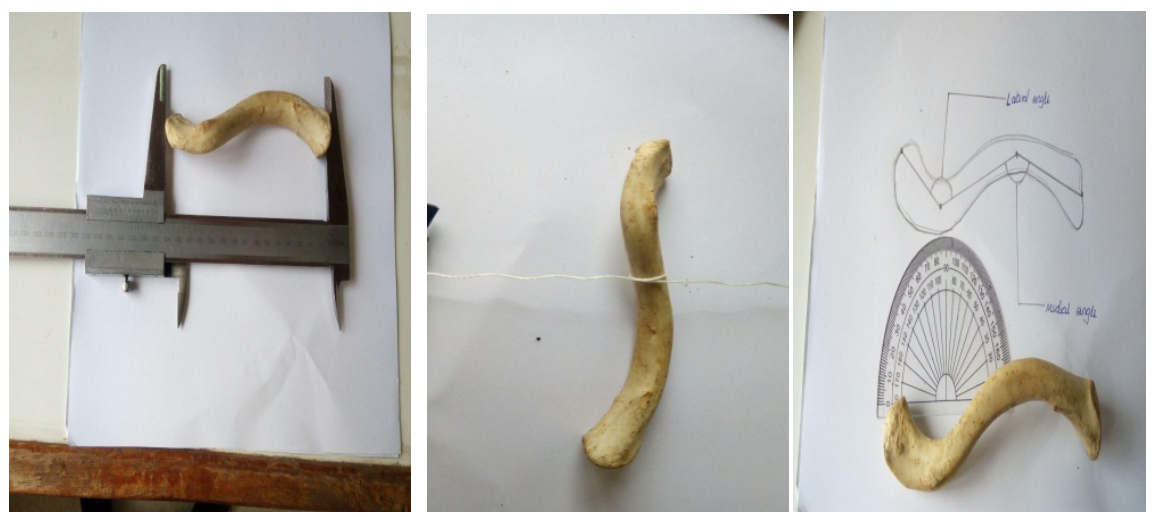

Figure 1: Image showing how the measurements were made using a Vernier caliper and a goniometer.

\section{RESULTS}

A total of 81 dried and cleaned adult clavicles of unknown sex were studied by measuring their length, medial angle of curvature, lateral angle of curvature and measuring midclavicular circumference, the results were as follows. Total of $42(51.9 \%)$ right clavicles and $39(48.1 \%)$ left clavicles were collected and used in this study. It was found that $12(14.8 \%)$ clavicles out of 81 clavicles were of lowest length $(<14 \mathrm{~cm}), 46$ (56.8\%) clavicles had medium length $(14-16) \mathrm{cm}$ and $23(28.4 \%)$ clavicles had highest length $(>16 \mathrm{~cm})$. There were $12(14.8 \%)$ clavicles with smallest lateral angle of curvature $<129^{\circ}$, $57(70.4 \%)$ clavicles had medium lateral angle of curvature (129-145) ${ }^{\circ}$ and $12(14.8 \%)$ clavicles had largest lateral angle of curvature $>145^{\circ}$. Forty seven $(58.0 \%)$ clavicles had medium medial angle of curvature (150-158) ${ }^{\circ}$, $22(27.2 \%)$ had smallest medial angle of curvature $<150^{\circ}$ and $12(14.8 \%)$ clavicles had largest medial angle of curvature $>158^{\circ}$. The lowest mid clavicular circumference $<3.6 \mathrm{~cm}$ had $16(19.8 \%)$ clavicles, the medium mid clavicular circumference (3.6-4.2) $\mathrm{cm}$ had 58 (71.6\%) clavicles and the highest mid clavicular circumference $>4.2 \mathrm{~cm}$ had $7(8.6 \%)$ clavicles.

The average length of left clavicle was $15.23 \pm$ $1.12 \mathrm{~cm}$ and the average length of right clavicle was $15.43 \pm 1.01 \mathrm{~cm}$. The right clavicle was slightly longer than the left clavicle, the difference was statistically insignificant $(p=0.37)$. The range of medial angle of curvature of left clavicle was $146^{\circ}$ to $164^{\circ}$ and that of right clavicle was $145^{\circ}$ to $162^{\circ}$. The average medial angle of curvature of left clavicle was $155.33^{\circ} \pm 4.39^{\circ}$ and that of right clavicle was $153.40^{\circ} \pm 3.96^{\circ}$. The medial angle of curvature of left clavicle was greater than medial angle of right clavicle, the difference was statistically significant because $p$-value was 0.004 . The average lateral angle of curvature of left clavicle was more than the average lateral angle of curvature of right clavicle. This difference was statistically insignificant because $(p=0.35)$. Total angle of curvature of left clavicle varied from $268^{\circ}$ to $316^{\circ}$ and the mean total angle of curvature was $293.54^{\circ} \pm 9.55^{\circ}$. Total angle of curvature of right clavicle varied from $268^{\circ}$ to $303^{\circ}$ and the average total angle of curvature $290.05^{\circ} \pm 8.94^{\circ}$. The range of midclavicular circumference of left clavicle varied from $3.1 \mathrm{~cm}$ to $4.4 \mathrm{~cm}$ and the average midclavicular circumference was $3.88 \mathrm{~cm} \pm 0.33 \mathrm{~cm}$. The mean of midclavicular circumference of right clavicle was $3.94 \mathrm{~cm} \pm$ $0.33 \mathrm{~cm}$ while its range was $2.9 \mathrm{~cm}$ to $4.6 \mathrm{~cm}$. 
Table 1. Comparison of parameters of clavicle

\begin{tabular}{|c|c|c|c|}
\hline \multicolumn{4}{|c|}{ Comparison of data of length of the clavicle: } \\
\hline Data & Left clavicles & Right clavicle & All clavicles \\
\hline Sample size & 39 & 42 & 81 \\
\hline mean \pm sd & $15.23 \pm 1.12$ & $15.43 \pm 1.01$ & $15.33 \pm 1.06$ \\
\hline Range & $12.74-17.61$ & $13.50-17.55$ & $12.74-17.61$ \\
\hline p-value & \multicolumn{2}{|l|}{0.37} & \\
\hline \multicolumn{4}{|c|}{ Comparison of data of medial angle of curvature: } \\
\hline Data & \begin{tabular}{|l} 
Left clavicle \\
\end{tabular} & Right clavicle & All clavicle \\
\hline Sample size & 39 & 42 & 81 \\
\hline mean \pm sd & $155.33 \pm 4.39$ & $153.40 \pm 3.96$ & $154.33 \pm 4.26$ \\
\hline Range & $146-164$ & $145-162$ & $145-164$ \\
\hline p-value & \multicolumn{2}{|l|}{0.04} & \\
\hline \multicolumn{4}{|c|}{ Comparison of data of lateral angle of curvature: } \\
\hline Data & Left clavicle & Right clavicle & All clavicle \\
\hline Sample size & 39 & 42 & 81 \\
\hline mean \pm sd & $138.26 \pm 8.21$ & $136.64 \pm 7.63$ & $137.42 \pm 7.91$ \\
\hline Range & $118-156$ & $118-148$ & $118-156$ \\
\hline $\mathrm{p}$-value & \multicolumn{2}{|l|}{0.35} & \\
\hline \multicolumn{4}{|c|}{ Comparison of data of midclavicular circumferences: } \\
\hline Data & Left clavicle & Right clavicle & All clavicle \\
\hline Sample size & 39 & 42 & 81 \\
\hline mean \pm sd & $3.88 \pm 0.33$ & $3.94 \pm 0.33$ & $3.91 \pm 0.33$ \\
\hline Range & $3.1-4.4$ & $2.9-4.6$ & $2.9-4.6$ \\
\hline p-value & \multicolumn{2}{|l|}{0.37} & \\
\hline
\end{tabular}

\section{DISCUSSION:}

In the present study, mean length of clavicle was $15.33 \mathrm{~cm}$ and range of clavicular length was $12.74 \mathrm{~cm}$ to $17.61 \mathrm{~cm}$. This is almost similar to the study done in South Africa which showed that the average length of the clavicle was 15.15 $\mathrm{cm}$ and range was $12.15 \mathrm{~cm}$ to $18.33 \mathrm{~cm}$ (King et al., 2014). This study also showed that the mean length of right clavicle $(15.43 \mathrm{~cm})$ was greater than the mean length of left clavicle $(15.23 \mathrm{~cm})$, this is similar to the study done in Telangana in which the mean length of the left clavicles was $137.83 \mathrm{~mm} \pm 7.99 \mathrm{~mm}$ and the mean length of the right clavicles was $138.71 \mathrm{~mm} \pm 8.66 \mathrm{~mm}$, (Kamdi et al., 2014). The present study showed that the average medial angle of curvature of left clavicle was $155.33^{\circ} \pm 4.39^{\circ}$ and that of right clavicle was $153.40^{\circ} \pm 3.96^{\circ}$. The medial angle of curvature of left clavicle was greater than medial angle of right clavicle, the difference was statistically significant because its $p$-value was 0.04 . This is similar to the study done in Nepal showed that the mean of the medial angle of the right clavicle was $150.970 \pm 6.16^{\circ}$ and the mean of the medial angle of the left clavicle was $151.50^{\circ} \pm 5.67^{\circ}$. The side differences were not statistically significant because the $p$-value was 0.47 The average lateral angle of curvature of left clavicle was $138.26^{\circ} \pm 8.21^{\circ}$ and this was more than the average lateral angle of curvature of right clavicle $136.64^{\circ} \pm 7.63^{\circ}$ this difference 
was statistically insignificant because $p$-value was 0.35 . This is consistent to study done in Nepal which reported that the mean of the lateral angle of the right clavicle was $139.76^{\circ}$ $\pm 7.55^{\circ}$ and the mean of the lateral angle of the left clavicle was $141.73^{\circ} \pm 8.44^{\circ}$. As the lateral angle of the left clavicle was greater than that of the right clavicle, it was showed that the lateral one-third of the clavicle on the left side was longer than the right side.

According to this study, it was reported that the range of midclavicular circumference of left clavicle varied from $3.1 \mathrm{~cm}$ to $4.4 \mathrm{~cm}$ and the average midclavicular circumference was 3.88 $\mathrm{cm} \pm 0.33 \mathrm{~cm}$. The mean of midclavicular circumference of right clavicle was $3.94 \mathrm{~cm} \pm$ $0.33 \mathrm{~cm}$ while its range was $2.9 \mathrm{~cm}$ to $4.6 \mathrm{~cm}$. No study has been done to establish the average midclavicular circumference of both genders. However, this is consistent to the study done in Gujarat zone which reported that the mid clavicular circumference of right clavicle for male varied from $3.0 \mathrm{~cm}$ to $4.6 \mathrm{~cm}$ with a mean of
$3.71 \mathrm{~cm} \pm 0.3 \mathrm{~cm}$. The left clavicle midclavicular circumference of male varies from $3.0 \mathrm{~cm}$ to $4.9 \mathrm{~cm}$ with a mean of $3.64 \mathrm{~cm} \pm 0.35 \mathrm{~cm}$. The mean of midclavicular circumference of right clavicle was greater than that of left clavicle.

In conclusion, the right clavicle is longer than the left clavicle, the average medial angle of curvature of left clavicle was greater than medial angle of right clavicle, the average lateral angle of curvature of left clavicle was more than the average lateral angle of curvature of right clavicle, the sum of the two angles of curvature was more on the left than on the right side and the mean of midclavicular circumference of right clavicle was greater than that of left clavicle. This variation could be due to racial, genetic or mechanical factors. The orthopedicians should consider these variations as it may be useful during surgical correction procedure of clavicle fracture and further studies should be done involving large sample sizes.

\section{COMPETING INTEREST: None}

ACKNOWLEDGEMENTS: I would like to express my sincere gratitude to my supervisor Dr D. A. Russa of department of anatomy of MUHAS for his assistance during my elective research work. His constructive ideas, moral and material support was helpful to me. Also, I should thank Dr. George Lufukuja of department of anatomy of HKMU who helped me during data collection; his assistance helped me to do this work.

\section{REFERENCES}

1. Aira JR. 2017. Research Article. doi: 10.1002/jor.23533.

2. Alcina M. 2015. Sexual dimorphism of the clavicle in a modern Spanish sample. 19(1): 73-83.

3. Bernat A. 2014. The Anatomy of the Clavicle: A Three-dimensional Cadaveric Study', 723(June 2012): 712-723. doi: 10.1002/ca.22288.

4. Hillen RJ, Bolsterlee B, Veeger DHEJ. 2016. Clinical Biomechanics The biomechanical effect of clavicular shortening on shoulder muscle function, a simulation study. JCLB. Elsevier Ltd, 37: 141-146. doi: 10.1016/j.clinbiomech.2016.07.005.

5. Ishwarkumar S. 2016. Sex Determination Using Morphometric and Morphological Dimensions of the Clavicle within the KwaZulu-Natal Population Determinación de Sexo dentro de la Población KwaZulu-Natal Mediante el Uso de Dimensiones Morfométricas y Morfológicas de la Clavícula', Int. J. Morphol, 34(1): 244-251.

6. Kamdi A. 2014. Morphometric Parameters and Sex Determination of Clavicle In Telangana 
Region', 13(10): 1-5.

7. Kaur H, Harjeet Sahni D. 2002. Length And Curves Of The Clavicle In Northwest Indians', J Anat. Soc. India, 51(2), pp. 199-209.

8. King PR, Scheepers S, Ikram A. 2014. Anatomy of the clavicle and its medullary canal: A computed tomography study', European Journal of Orthopaedic Surgery and Traumatology. doi: 10.1007/s00590-012-1130-9.

9. Liu P. 2010. Minimally Invasive Fixation of Displaced Midclavicular Fractures With Titanium Elastic Nails', 24(4): 217-223.

10. Qiu XS. 2016. Anatomical study of the clavicles in a Chinese population', BioMed Research International. doi: 10.1155/2016/6219761.

11. Rathna B. 2015. Variability of length and Osteometrical study of human clavicle and its applied importance', Scholars Academic Journal of BiosciencesOnline) Sch. Acad. J. Biosci, 3(9). Available at: www.saspublisher.com. 